\title{
MicroRNAs as fine-tuners of gene regulation in plant-microbe interactions
}

\author{
Minu M. Nair and Alagu Manickavelu* \\ Department of Genomic Science, Central University of Kerala, Kasargod 671 316, India
}

\begin{abstract}
Plants utilize non-coding RNA-mediated gene regulatory mechanism as one of the effective ways to cope with stress. MicroRNAs (miRNAs) are crucial players of this category that regulate gene expressions at the post-transcriptional level. miRNAs are reported to be involved in different aspects of plant-microbe interactions, starting from regulation of pathogen recognition receptors to fine regulation of defence gene expression, as well as in maintaining symbiosis. In plants, miRNA mediated gene regulation is an active layer in biotic stress responses with a lesser chance of off-target effects. A better understanding of miRNAmediated gene regulation will have numerous applications from primary gene function study to targeted gene silencing. This article summarizes the current knowledge on miRNAs in plant-microbe interactions, with an emphasis on their utilization as a powerful tool in disease management.
\end{abstract}

Keywords: Disease management, gene regulation, microRNAs, plant-microbe interactions, plant defence responses.

PLANTS are the mainstay for survival of other organisms on earth and maintain harmony of the biosphere. They are considered as a major source to feed the entire world population. The increase in global population, as well as various abiotic and biotic stresses challenge plant protection and food security. Plant-microbe interactions have been a focal point of research since a long time, as they can change the morphological, physiological and metabolic processes of the host plants. Interactions between plants and microbes could either be beneficial or detrimental. Mutualistic interactions can impart better adaptation, while interactions with pathogenic microbes result in devastating plant diseases ${ }^{1}$. The pathogenic intrusion triggers the plant immune system and the recognition of pathogen/microbe-associated molecular patterns (PAMPs/ MAMPs) using pattern recognition receptors (PRRs) as the first response $\mathrm{e}^{2,3}$. This initiates production of reactive oxygen species (ROS), callose deposition to strengthen the cell wall, expression of pathogenesis-related (PR) genes and production of phytohormones. Collectively, these responses are classified as PAMP-triggered immun-

\footnotetext{
*For correspondence. (e-mail: amanicks@cukerala.ac.in)
}

ity $(\mathrm{PTI})^{4}$. Nevertheless, the pathogens have evolved to take over these primary responses of the host via effector proteins, which compel the host plants to establish effector-triggered immunity (ETI) as another active layer of immune response. ETI responses are mainly via the expression of resistance genes which lead to hypersensitive responses upon recognition of effector proteins produced by the pathogens 5 .

Emergence of new microbial mechanisms to invade the host plant and constant exposure to the pathogens encourage the plants to develop more efficient and sophisticated mechanisms to control pathogen attack. One such mode that provided new dimensions to the complex eukaryotic gene regulatory mechanisms is non-coding RNA-mediated gene regulation. The major contributors to this category are microRNAs (miRNAs), and their role in different aspects of growth, development, chromatin remodelling, genome stability and stress responses has been reported ${ }^{6,7}$.

miRNAs are single-stranded RNAs of $18-25 \mathrm{nt}$ in length, and regulate the genes post-transcriptionally either through mRNA cleavage or by translational repression based on their complementarity with the target mRNAs ${ }^{8}$. The miRNAs were initially known as short temporal RNAs (stRNAs) and first reported in Caenorhabditis elegans by early 1990s, but several plant miRNAs have been discovered later ${ }^{9-12}$. The biogenesis of miRNAs in plants is well studied (Figure 1). miRNA synthesis starts from the transcription of MIR genes by RNA polymerase II, and proceeds through the formation of primary miRNAs, precursor miRNAs, miRNAmiRNA* duplex before single-stranded miRNAs get inserted into the RNA-induced silencing complex (RISC). Following the RISC formation, ARGONAUTE (AGO) proteins guide the miRNA strand to its target mRNA, and other strand of the duplex undergoes degradation ${ }^{13-23}$.

The precise and targeted gene regulation makes plant miRNAs distinct and efficient gene modulators. A better understanding of miRNAs can be exploited for its myriads of applications from basic gene function study to targeted gene silencing ${ }^{24}$. By taking advantage of sequence specificity of endogenous miRNAs, designing of artificial miRNAs to suppress the target genes for enhancement of quality and quantity of agricultural products is also in practice ${ }^{25}$. Efforts have been made in the past for the identification of many miRNA-mRNA 
modules in plant-microbe interactions, but very few have been functionally characterized and validated for agronomic importance ${ }^{26}$. Individual miRNAs could play distinct roles through different targets in discrete conditions and across various species ${ }^{27}$. So the accurate identification of miRNAs as gene modulators with efficacy and reliability is essential for their utilization in genetic manipulation of crops. A cumulative approach using bioinformatic tools and biological experiments will help identify the correct candidate miRNAs. In this article, we summarize the advances in miRNA-mediated regulation of genes related to plant-microbe interactions (Figure 2), with an emphasis on the role of plant miRNAs in disease susceptibility and resistance, which can be exploited for growing improved crop varieties.

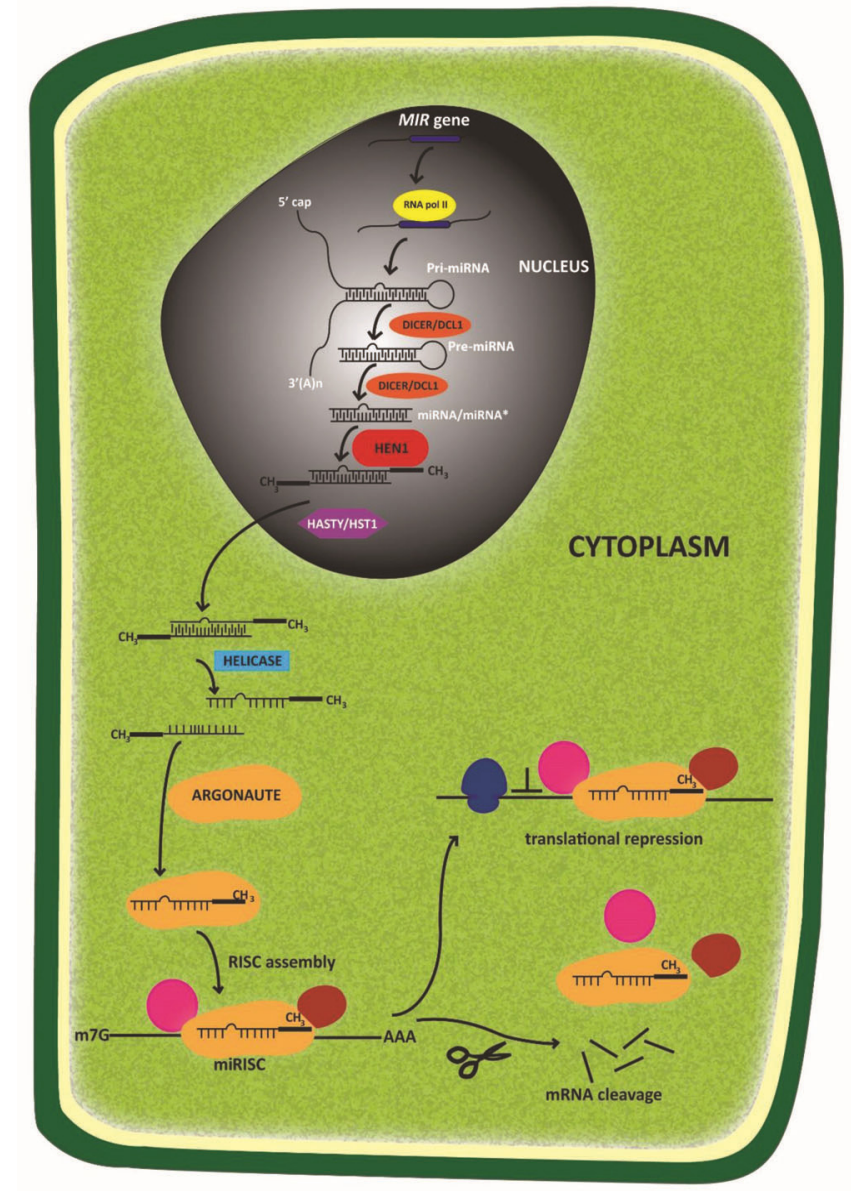

Figure 1. MicroRNA biogenesis and mode of action in plants. miRNAs are transcribed from $M I R$ genes by RNA polymerase-II and the initial product is known as primary miRNA/pri-miRNA. The pri-miRNA undergoes two-step cleavage mediated by DICER/ DCL1 resulting in an intermediate known as precursor miRNA/premiRNA, followed by the formation of miRNA-miRNA* duplex. This duplex undergoes methylation in the nucleus mediated by HEN1 protein. Later it is transported to the cytoplasm with the help of HASTY/HST1 protein and the strands of the duplex are separated by helicase. The miRNA strands are incorporated into the RISC assembly with the aid of ARGONAUTE proteins. The RISC assembly can either direct mRNA cleavage or translational repression of mRNAs based on the complementarity between miRNA and its target mRNA.

\section{MicroRNAs as cardinal regulators in mutualistic plant-microbe interactions}

Mutualistic interactions couple different species that live in proximity for reciprocal favours. This is more prevalent between plants and the organisms that live inside the plants. These mutualistic endophytes help the host plants in growth, development, fitness, adaptation to environmental conditions and in combating pathogen infection. Though the mechanism and molecular interplay driving the symbiotic plant-microbe interactions are well established, only few studies have been focused on the regulatory part of these interactions, especially through miRNA mode. Case studies related to the most common mutualistic interactions are described here. Root nodule (RN) symbiosis and arbuscular mycorrhizal symbiosis are the most common and well-studied mutualistic plantmicrobe systems ${ }^{28,29}$.

The RN symbiosis, typically associated with legumes, is considered as one of the best reported plant-endophytic mutual relationships. It is well known for nitrogen fixation by nitrogen-fixing bacteria inside specialized organs known as root nodules in the plant ${ }^{30}$. In the context of RN symbiosis, miRNAs are outlined as the molecular signatures in the regulatory network of nodule development and nutrient homeostasis ${ }^{31}$. One of the well-known micro-regulators is miR169 that modulates root nodule development and meristematic activity. In Medicago truncatula miR169a has been identified to target a CCAAT-binding family of transcription factors known as Heme Activator Protein 2-1 (MtHAP2-1) and confines the protein expression in nodule meristematic zone $\mathrm{e}^{32}$. It is proposed that miR169a mediated regulation of MtHAP21 may be essential for nodule cell differentiation, since the gene is required for meristematic activity and maintenance. Likewise, miR166 of $M$. truncatula targets a class-III homeodomain-leucine zipper (HD-ZIP III) family of transcription factors which are associated with nodule development. The study revealed that miR166 co-expressed with its target transcription factors in vascular bundles, apical regions of roots and nodules causing a reduction in nodule number and lateral roots ${ }^{33}$. In soybean, Subramanian et al. $^{34}$ reported the temporal differential expression of miRNAs in response to inoculation with Bradyrhizobium japonicum. In order to determine the regulatory roles played by the differentially regulated miRNAs, they observed that miR169 has two putative targets in soybean which are identical to HAP2-1 of $M$. truncatula which has already been reported for nodule development. Besides, they have identified that miR172, miR166 and miR396 regulate a putative Apetala2-like transcription factor, HD-ZIP III-like transcription factor, and a cysteine protease respectively ${ }^{34}$. Few of the reported miRNAs are linked to plant hormone auxin signalling and its homeostasis, which might be an essential bridge for nodule development. In this category, 


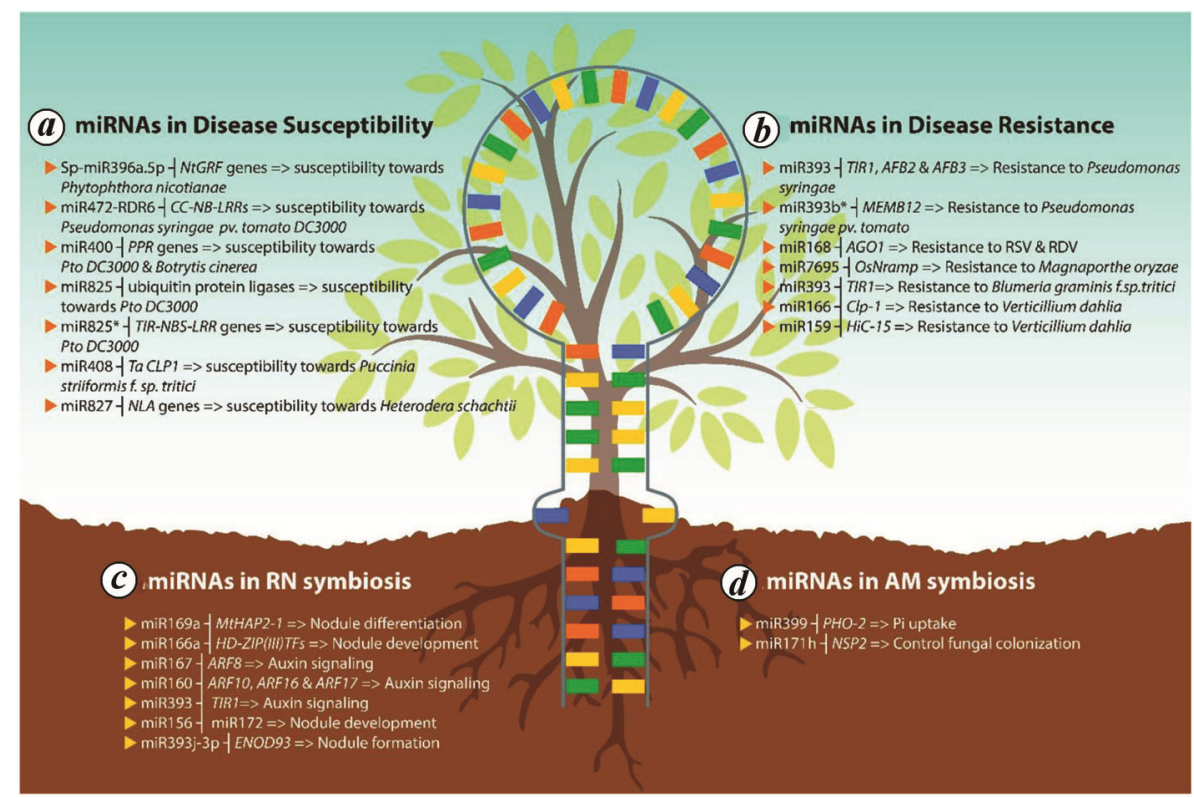

Figure 2. Overview of miRNAs present in plant-microbe interactions. $\boldsymbol{a}$, Plant miRNAs and their corresponding targets involved in increasing host susceptibility towards pathogen infection. $\boldsymbol{b}$, Plant miRNAs and their corresponding targets involved in increasing resistance responses towards pathogen infection. $\boldsymbol{c}$, Plant miRNAs and their corresponding targets having a role in root nodule symbiosis. $\boldsymbol{d}$, Plant miRNAs and their corresponding targets having a role in arbuscular mycorrhizal symbiosis.

miR167 targets auxin response activator ARF8, miR160 regulates auxin response repressors ARF10, 16 and 17, miR393 targets an auxin receptor TIR1 and miR164 regulates a NAC1 transcription factor ${ }^{8,35,36}$. The role of auxin and auxin signalling during root nodule formation was identified through various studies. Local auxin accumulation was reported as an important requisite for nodule organogenesis ${ }^{37}$. In addition, endogenous application of an auxin signalling regulator, IAA7, led to increased nodulation in Casuarina glauca $^{38}$. These reports also validate the role of auxin signalling during RN symbiosis. Moreover, ectopic expression of miR172 of soybean increased the number of nodules, expression of symbiotic leghemoglobin and non-symbiotic haemoglobin by limiting the level of an AP2 transcription factor. miR156 was found to negatively regulate the expression of miR172 (ref. 39). So, the authors postulated that the antagonistic effects of miR156 and miR172 control nodule development in soybean. Likewise, miR172c-mediated regulation of AP2-1 was shown to be involved in common bean (Phaseolus vulgaris) - Rhizobium etli symbiosis. AP2-1 acts as a transcriptional activator of several other genes related to nodule senescence as well. The authors proposed a model in which these genes got silenced by miR172c-induced AP2-1 cleavage. The positive regulator role of miR172c in this interaction was validated with the overexpression of miRNAs, where magnification in rhizobial infection, nodule formation, nitrogen fixation, etc. was observed ${ }^{40}$. Similarly, ectopic expression of miR160 resulted in auxin hypersensitivity and cytokinin hyposensitivity, which cause moderate-level inhibition of primordium formation and inhibition of further development of nodules ${ }^{41}$. In addition, recently miR393j-3p was identified as a potential regulator of nodule formation in soybean, by targeting a nodulin gene known as ENOD93 (Early Nodulin 93), and its ectopic expression resulted in a significant reduction in the number of nodules $^{42}$. Many more miRNA repertoires have been reported in association with the legume-rhizobia symbiosis, but few have focused on the roles played by specific miRNAs.

The symbiotic association between most of the plants and glomeromycotian fungi is commonly known as arbuscular mycorrhizal (AM) symbiosis, which enhances phosphate availability to the plant partner and also improves plant resistance towards abiotic and biotic stresses $^{29}$. The crucial role of miRNAs in the modulation of AM symbiosis has been identified in recent studies. miR399 has been reported in the context of AM symbiosis in M. truncatula as well as in Nicotiana tabacum plants. Studies have revealed that AM symbiosis leads to increased expression of miR399, but not as a good signal for improving mycorrhizal colonization. Instead, it acts as a modulator for sufficient phosphate uptake during AM symbiosis by targeting the transcript of a ubiquitinconjugating enzyme PHO-2, which is a negative regulator of $\mathrm{Pi}$ starvation inducible genes ${ }^{43}$. Another important finding was that both miRNAs and miRNA*s are differentially expressed during AM symbiosis, which may indicate their involvement in the process. Several of these miRNAs and miRNA*s target disease resistance genes for promoting fungal growth ${ }^{44}$. Interestingly, studies 
revealed that miRNAs prevent the over-colonization of symbiotic fungi around plant roots. miR171h of M. truncatula negatively regulates NSP2, a transcription factor involved in both nodulation and mycorrhization. Since NSP2 is vital for root colonization, miR171h-mediated transcriptional regulation of NSP2 is considered as a mechanism to prevent over-colonization, or to spatially limit the fungal growth ${ }^{45}$. A recent study has reported six AM symbiosis-related miRNAs in tomato, and two of these miRNAs belong to the miR171 family that are capable of targeting the NSP2 genes ${ }^{46}$. These studies suggest that regulation of NSP 2 genes mediated by the miR 171 family serves as a general strategy in the regulation of AM symbiosis. Unlike other members of the miR171 family, miR171b promotes the establishment of AM symbiosis. miR171b has a mismatched cleavage site among plants that establish AM symbiosis, so that it is unable to target LOST MERISTEMS1 (LOM1) gene, which is the regular target of the miR171 family during AM symbiosis ${ }^{47}$. This clearly shows the antagonistic act of miRNAs from the same miRNA family for maintaining the balance of one biological function. Except for these established mutualistic relations, miRNA-mediated regulation befitting in other plant-endophyte relations are sparse. A study on the aforementioned group was the identification of differentially expressed miRNAs and their putative targets in Oncidium orchid during the infection of an endophytic fungi Piriformospora indica. These miRNAs were reported to regulate development, perception of auxin signalling, plant defence, etc. ${ }^{48}$.

Regulation of mutualistic interactions through miRNAs is a vast area of research with immense potential, but less exploited. Though miRNAs are identified as a promising modulator for improving the effectiveness of mutualistic relations in terms of plant mineral nutrition, there are hardly any studies focusing on miRNA-mediated improvement of association efficacy. Functional analysis of modified miRNA expression can ensure novel insights for enhancing plant mineral nutrition through the symbiotic plant-microbe interactions.

\section{MicroRNAs and plant disease susceptibility}

Plant-microbe interactions could also be hostile and result in the development of various plant diseases. When it comes to plant diseases, host susceptibility is equally important as host resistance. However it has not been a focal point of research studies in the past. Earlier studies reported only the inactivation of host miRNAs by the ingenious act of pathogen as a part of their counter defence programming ${ }^{49}$. Whereas, recent developments with scientific evidence exhibit the pathogen-induced expression profile of host miRNAs and downstream pathways in initiating susceptibility. An important discovery was the dual phenotype of transgenic tobacco plants with overexpressed Sp-miR396a-5p. The transgenic plants showed resistance for various abiotic stresses like salt, cold and drought stress in contrast with their increased susceptibility towards Phytophthora nicotianae infection. The contrary roles in various stresses were due to gene regulation of members from the NtGRF family ${ }^{50}$. This case itself shows the importance of understanding the functions of single miRNAs at various stresses before their application. siRNAs generated in Arabidopsis by the merged operation of miR472 and RNA-dependent RNA polymerase 6 (RDR6), silence the resistance genes of the coiled-coil nucleotide-binding leucine rich-repeats (CC$N B-L R R s / C N L s$ ) family. The results have been validated through loss- and gain-of-function studies. Loss-offunction lines for both miR472 and RDR6 were resistant towards Pseudomonas syringae pv. tomato DC3000 (Pto DC3000) with effector AvrPphB, whereas the overexpression lines showed susceptibility ${ }^{51}$. Though this study showed the increased susceptibility due to miR472RDR6-mediated silencing of basal defence responses, the possibility to get infected by other pathogens in this immune-compromised condition was not reported.

In a similar study, transgenic Arabidopsis plants that overexpress miR400 exhibited a significant increase in vulnerability towards both bacterium Pto DC3000 and the fungus Botrytis cinerea. It was found and validated that negative regulation of pentatricopeptide $(P P R)$ genes by miRNA400 contributed to the phenotypes ${ }^{52}$. Another study in Arabidopsis revealed the role of both miR825 and miR825* from the duplex in enhancing susceptibility towards Pto DC3000 with entirely different classes of genes as targets. miR825 regulates ubiquitin-protein ligases, whereas miR825* regulates toll-interleukin-like receptor-nucleotide-binding site-leucine-rich repeat (TIRNBS-LRR) type R genes, a component of resistance responses. A strong validation was obtained when the pretreatment with plant growth-promoting rhizobacterium Bacillus cereus AR156 was found to suppress the activity of miR825 and miR825* and resulted in induced systemic resistance (ISR) against Pto DC3000 (ref. 53). The same group has recently reported the priming of AR156elicited systemic resistance to Botrytis cinerea by suppressing miR825 and miR825* (ref. 54). These results indicate the possibility of utilizing $B$. cereus AR156 as a control machinery against infection. Recently, it has been reported that there are wheat-specific miRNAs involved in susceptible interaction with Puccinia striiformis f. sp. tritici, causing stripe rust in wheat. The preliminary bioinformatic study has found potential miRNAs and their downregulated targets having a role in causing the susceptibility interaction between the host plant and pathogen; however, further biological characterization is necessary for authentication ${ }^{55}$. Another study found that stripe rust infection in wheat primes miR408-mediated susceptibility towards the disease via the regulation of TaCLPl gene. The gene encodes for a chemocyanin-like 
protein, essential for mediating resistance response to salinity stress and high cupric content ${ }^{56}$. Evidence suggests that plant miRNAs can also make the host susceptible to parasitic infections. A case in point is miR827 of Arabidopsis, that enhances the vulnerability for a sedentary endoparasite, beet cyst nematode Heterodera schachtii. miR827 modulates nitrogen limitation adaptation $(N L A)$ genes that encode ubiquitin E3 ligase enzyme required to limit the activity of parasites. Moreover, inactivation of miR827 reduces susceptibility and overexpression leads to hypersusceptibility towards the nematode, which validates the findings ${ }^{57}$. Disabling the host miRNAs, essential for mediating defence responses, is another pathogen mechanism to increase disease susceptibility. For example, during the interaction between susceptible poplar plant and virulent Melampsora larici-populina, miRNAs of defence-signalling pathways got inactivated. Taking control over these miRNAs was part of the effector-triggered susceptibility of pathogens ${ }^{58}$.

Inducing the production of host miRNAs that can control the defence response is a general strategy of pathogens to make the host plant susceptible. Identifying such miRNAs and their mode of action has a potential role in disease management. Furthermore, production of genetically modified plants that hinder the activity of these candidate miRNAs upon infections can effectively control disease outbreaks and thereby reduce loss in crop productivity.

\section{MiRNAs as a defence machinery in plant-pathogen interactions}

Plants utilize a broad range of defence mechanisms in order to a control pathogen infection. In this context, microRNAs have been reported to foster plant resistance response upon biotic stress, irrespective of the causative organism $^{59}$. The influence of plant miRNAs in resistance response is relatively well studied compared to their role in disease susceptibility and symbiotic associations, due to their vast potentiality in raising disease-resistant varieties. Modulation of genes that promote invasion and growth of pathogens inside the host is the typical manner by which miRNAs aid plant immune responses. The various miRNA-associated pathways ward to tackle the dangers associated with pathogen attacks are discussed here.

The first and foremost report on a miRNA with its role in pathogenic resistance was miR393 of Arabidopsis, that enhanced resistance against bacterium Pseudomonas syringae infection. In this report, miR393 which induced upon treatment with flg22 (a flagellin derived peptide) was found to negatively regulate the transcripts of auxin receptor F-box genes such as TIR1, AFB2 and AFB3. This resistance to bacterial infection through the suppression of auxin signalling pathways by miR393 was further confirmed by gain-of-function studies ${ }^{60}$. The invaded pathogens modulate host auxin signalling to antagonize the salicylic acid (SA)-dependent responses. So, by targeting the components of auxin signalling, miR393 hinders SA pathway suppression and thus its involvement in activating SA pathway for defensive responses against $P$. syringae has been established. A model to prioritize supplies for defence responses by miR393 has been proposed, where it increases the production of antimicrobial compound glucosinolate and redirects the secondary metabolite biosynthesis via decreasing camalexin level ${ }^{61}$. Similarly, miR393-mediated repression of auxin receptors has been reported in other plants too. In rice, miR393 targets OsTIR1 and OsAFB2 and several studies have documented the other phenotypes of miR393 overexpression lines such as the increased inclination of flag leaves at booting stage, altered primary and crown root growth, increased tillers, early flowering and reduced tolerance to drought and salt stress ${ }^{62,63}$. Interestingly, miR393 was produced upon the infiltration of oncogenic strains of Agrobacterium tumefaciens, whereas the non-oncogenic strains could not trigger the production. It suggests the possible role of miR393-mediated immune responses to the oncogenic strains ${ }^{64}$. Apart from miR393, miR393* of Arabidopsis has also proved its capacity in antibacterial immunity. The aforementioned study also reports that miR393b* targets a Golgi-localized SNARE gene, $M E M B 12$ that encodes a protein required for membrane fusion. Here, the downregulation of $M E M B 12$ increases exocytosis of antimicrobial pathogenesis-related protein, PR1 and enhances resistance towards $P$. syringae pv. tomato $(P s t)^{65}$.

Along with miR393, miR160 and miR167 were also found to enhance the PTI by targeting transcription factors of the auxin response factor (ARF) family, which is a good mechanism in limiting the growth of $P$. syrin$g_{a} e^{66}$. Arabidopsis overexpression lines for miR160a, showed improved resistance response and higher callose deposition by modulating ARFs, whereas overexpression of miR398 and miR773 enhanced susceptibility to both virulent and non-virulent strains of $P$. syringae by regulating PAMP-induced callose deposition. This antagonistic response is mediated by different signalling pathways according to the pathogen as well as environmental conditions. miR160a is a positive regulator of PAMP-induced callose deposition, whereas miR398b and miR773 are negative regulators of the same ${ }^{67}$. Evidence revealed that miR398 targets $\mathrm{Cu} / \mathrm{Zn}$ superoxide dismutase 1 and 2 (CSD1 and CSD2), the group of metalloenzymes and scavengers of the ROS in Arabidopsis. Also, miR398 was downregulated during oxidative stress and infection from virulent strains of Pst, in pursuance of preventing negative regulation on ROS production, which is a requisite for basal defence mechanism in PTI and stress respon$\operatorname{ses}^{68,69}$. This represents another strategy of the hosts in which miRNAs that control basal responses itself are under tight regulation for the prevention of disease spread. 
Non-coding RNA-mediated gene silencing mechanism is a recurrent strategy of resistance response against viral infections in plants. Studies have implicated the lucid role of miRNAs as a critical layer of antiviral defence. In Brassica rapa, the infection by Turnip mosaic virus (TuMV) induces production of bra-miR1885, and it targets a Toll/interleukin-1, a TIR-NBS-LRR resistance gene. The study reports bra-miR1885 for the first time as novel Brassica-specific miRNAs, and proposed that it may originate through duplication events in the TIRNBS-LRR class of genes based on sequence similarity between the miRNA precursor sequences and target sequences $^{70}$. In a comparative study where rice plants were infected with Rice stripe virus (RSV) and Rice dwarf virus (RDV), alteration in the induction of host small RNAs was observed in RSV infection only. RSV infection increased the accumulation of some miRNA*s instead of miRNAs, and the expression of some novel, phased miRNAs from conserved miRNAs ${ }^{71}$. Another discovery during RSV infection was the recently evolved positive regulatory mechanism of defence system by AGO18 protein. The antiviral protein AGO1 was found to be inhibited by miR168. In the course of infection, AGO18 sequester the miR168 which in turn magnifies AGO1-enabled antiviral activity ${ }^{72}$. The study points out the necessity for identification and utilization of proteins that confer broad-spectrum resistance conferred by these proteins. Several host miRNAs were found to target the NBS-LRR resistance genes and restraining the activity of these miRNAs is another antiviral safeguarding strategy of plants. For instance, a microRNA superfamily, including miR482 and miR2118, targets disease resistance protein NBS-LRR motifs. In Nicotiana benthamiana, miR482 initiates mRNA decay of NBS-LRR disease resistance proteins with coiled-coil domains and produces secondary siRNAs, which target mRNAs of other defence-related proteins. During infections of Turnip crinkle virus (TCV), Cucumber mosaic virus (CMV) and Tobacco rattle virus (TRV), miR482-mediated regulatory cascade gets inactivated, which leads to the activation of defence responses via downstream mRNA targets of miR482 (ref. 73). Targeted gene silencing via artificial miRNAs (amiRNAs) is a robust technology used in plants to suppress viral attack, in comparison to other pathogen infections. In 2012, researchers found success in developing transgenic wheat with five amiRNAs inside one polycistronic amiRNA precursor of rice miR395, named as FanGuard (FGmiR395), and providing resistance against Wheat streak mosaic virus (WSMV) ${ }^{25}$. Another critical development in this regard was the development of transgenic rice plants with designed dimeric amiRNA precursor expression vectors that resemble the precursor of rice miRNA, osa-miR528. These plants were found to be simultaneously resistant towards RSV and Rice black streaked dwarf virus (RBSDV). This was achieved by controlling the Coat protein genes (CP) of RSV and
$\mathrm{RBSDV}^{74}$. Utilization of such amiRNAs for conferring long-lasting and broad-spectrum virus resistance is one of the advancements that is being adopted.

The pivotal role of miRNAs has been explained in antifungal response also. In rice, miR160a and miR398b showed altered expression during Magnaporthe oryzae infection. Transgenic plants overexpressing these miRNAs exhibited increased $\mathrm{H}_{2} \mathrm{O}_{2}$ accumulation at the site of infection and enhanced the expression of defence-related genes such as PRI and PR10. These reactions helped the host in fighting against the infection ${ }^{75}$. Similarly, miR7695 of rice also conferred resistance against $M$. oryzae. Upon infection, expression of miR7695 increased and it controlled the production of an alternatively spliced transcript of Natural resistance-associated macrophage protein6 (OsNramp6) gene. The protein encoded by the target gene is part of the divalent metal ion transport system and a contributor of metal ion homeostasis. Overexpression of miR7695 has shown to boost the immune responses against infection by negatively regulating the metal-ion transport ${ }^{76}$. Restrictions on metal-ion transport is a host response in order to prevent its utilization for the growth of pathogens inside the host, and a study points out the role of miRNAs in this natural mechanism ${ }^{77}$. In wheat infected with Puccinia graminis f.sp. tritici, several miRNAs such as miR159, miR164, miR167, miR171, miR444, miR408, miR1129 and miR1138 showed altered expression. The probable involvement of these miRNAs in PTI is by regulating mRNAs of different genes of hormone signalling, lignin biosynthesis and protein biosynthesis pathways ${ }^{78}$. Among these, miR159, miR164, miR167, miR171 and miR444 target transcription factors like $M Y B, G A M Y B, N A C 1$, $A R F s, S C L$ and $M A D S$-box respectively, are involved in different hormone signalling pathways ${ }^{12,79}$. These results validate the part of miRNAs in taking control over the plant hormone signalling pathways to reduce pathogen infection. Besides, in wheat upon infection with Blumeria graminis f.sp. tritici (Egt), miR156 is downregulated, and miR393 is upregulated in the R-gene carrying line, compared to the susceptible line. The miR393 targets auxin-responsive factor TIR1, and thereby controlling auxin signalling for mediating defence response against Egt in wheat ${ }^{80}$. The role of the miR172 family in flowering time regulation is well studied, and its contributions in biotic stress responses have been revealed recently. miRNA target mining in wheat disease EST libraries of leaf rust, powdery mildew and blast disease showed an abundance of miRNA172 target sites $^{81}$. In addition, overexpression of miR172a and $b$ in tomato has provided improved resistance responses against Phytophthora infestans by controlling the expression of AP2/ERF transcription factors. A study showed that the regulation of $\mathrm{AP} 2 / \mathrm{ERF}$ transcription factor via miR172 enhances ROS detoxification and thereby prevents cellular damage associated with the infection ${ }^{82}$. An 


\section{REVIEW ARTICLES}

exciting finding in antifungal defence mechanism is the regulation of pathogen-encoded virulence genes by plant miRNAs. During Verticillium dahlia infection in cotton plants, miR166 and miR159 were found to target two virulence genes of the fungus, the $\mathrm{Ca}^{2+}$ dependent cysteine proteases $(\mathrm{Clp}-1)$ and isotrichodermin $C-15$ hydroxylase $(\mathrm{HiC}-15)$ respectively, and modulation over these genes prevented fungal invasion ${ }^{83}$. This is evidence for the cross-kingdom miRNA regulation, a poorly explored area, and identification of such plant miRNAs which can regulate the virulence of the pathogen genes has immense applications in crop improvement programmes for effective disease management.

miRNA-mediated gene silencing is a promising strategy for plants against pathogens attack. Though many plantmicrobe systems showed an altered profile of several miRNAs upon different stages of infection, the evident role of individual miRNAs is still unclear. In the era of genome editing, we can effectively use the wellcharacterized miRNAs to fight against the vast number of pathogens. Plant miRNAs which can ultimately provide broad-spectrum disease resistance and take control over pathogenic genes will be important in the near future.

\section{Conclusion}

Small non-coding RNA, specifically miRNA-mediated gene regulation, is a robust strategy developed in plants against pathogens as well as in the mutualistic interactions between plants and microbes. This is an important field of research considering the potential applications of miRNAs in crop breeding programmes for sustainable development and food security. Using advancements in sequencing technologies and extensive efforts, many such miRNAs have been identified and characterized in recent times. The extraordinary potentiality of miRNAs from necessary gene function study to targeted gene expression control makes them powerful tools in molecular biology. R-gene-mediated resistance has been established as a commonly used mechanism for providing disease resistance in plant breeding. Gradually, this mechanism fails due to the emergence of new pathotypes and the release of new effector molecules in existing pathotypes. So, miRNA-mediated target gene silencing is a promising strategy for developing disease-resistant varieties. Though these micro-regulators are endogenous in nature, the exploitation of these miRNAs in crop improvement should be done carefully, since the induction of miRNAs is specific to different conditions like various species and strains of plants and microbes, the infection stages, growth phase of plants, presence or absence of other interacting genes, nutritional status, etc. So rather than studying the differential expression status of miRNAs in different plant-microbe models, it is worthy to identify the key roles played by each miRNA in specific plant- microbe interactions, since the same miRNA can have several functions under different circumstances. Both conserved and novel miRNAs can be identified by combining bioinformatics and experimental approaches. Lossof-function and gain-of-function studies are important in order to find the effect of miRNAs in other phenotypes, and that can be done even with the latest gene-editing technologies such as CRISPR-Cas9 system. Inducing specific miRNAs upon a particular pathogenic infection is also possible by cloning binding sites for pathogenspecific effectors or pathogen recognition sites in the promoter of these genes so that the binding will enhance the expression of miRNA genes. Identification of plant miRNAs which can provide broad-spectrum disease resistance and miRNAs which target virulence genes of pathogens, and employing them for crop breeding will be instrumental in controlling disease outbreaks and yield loss. The miRNA-mediated crop breeding programmes will add new dimensions to improve agronomic traits.

1. Selosse, M.-A., Baudoin, E. and Vandenkoornhuyse, P., Symbiotic microorganisms, a key for ecological success and protection of plants. C. R. Biol., 2004, 327, 639-648.

2. Delaunois, B., Jeandet, P., Clément, C., Baillieul, F., Dorey, S. and Cordelier, S., Uncovering plant-pathogen crosstalk through apoplastic proteomic studies. Front. Plant Sci., 2014, 5, 249.

3. Trdá, L., Boutrot, F., Claverie, J., Brulé, D., Dorey, S. and Poinssot, B., Perception of pathogenic or beneficial bacteria and their evasion of host immunity: pattern recognition receptors in the frontline. Front. Plant Sci., 2015, 6, 219.

4. Chisholm, S. T., Coaker, G., Day, B. and Staskawicz, B. J., Hostmicrobe interactions: shaping the evolution of the plant immune response. Cell, 2006, 124, 803-814.

5. Kushalappa, A. C. and Gunnaiah, R., Metabolo-proteomics to discover plant biotic stress resistance genes. Trends Plant Sci., 2013, 18, 522-531.

6. Chen, X., Small RNAs and their roles in plant development. Annu. Rev. Cell Dev. Biol., 2009, 25, 21-44.

7. Sun, G., MicroRNAs and their diverse functions in plants. Plant Mol. Biol., 2012, 80, 17-36.

8. Rhoades, M. W., Reinhart, B. J., Lim, L. P., Burge, C. B., Bartel, B. and Bartel, D. P., Prediction of plant microRNA targets. Cell, 2002, 110, 513-520.

9. Lee, R. C., Feinbaum, R. L. and Ambros, V., The C. elegans heterochronic gene lin-4 encodes small RNAs with antisense complementarity to lin-14. Cell, 1993, 75, 843-854.

10. Llave, C., Kasschau, K. D., Rector, M. A. and Carrington, J. C., Endogenous and silencing-associated small RNAs in plants. Plant Cell, 2002, 14, 1605-1619.

11. Park, W., Li, J., Song, R., Messing, J. and Chen, X., CARPEL FACTORY, a Dicer homolog, and HEN1, a novel protein, act in microRNA metabolism in Arabidopsis thaliana. Curr. Biol., 2002, 12, 1484-1495.

12. Reinhart, B. J., Weinstein, E. G., Rhoades, M. W., Bartel, B. and Bartel, D. P., MicroRNAs in plants. Genes Dev., 2002, 16, 16161626.

13. Lee, Y., Jeon, K., Lee, J. T., Kim, S. and Kim, V. N., MicroRNA maturation: stepwise processing and subcellular localization. EMBO J., 2002, 21, 4663-4670.

14. Lee, Y., Kim, M., Han, J., Yeom, K. H., Lee, S., Baek, S. H. and Kim, V. N., MicroRNA genes are transcribed by RNA polymerase II. EMBO J., 2004, 23, 4051-4060. 
15. Schauer, S. E., Jacobsen, S. E., Meinke, D. W. and Ray, A. DICER-LIKE1: blind men and elephants in Arabidopsis development. Trends Plant Sci., 2002, 7, 487-491.

16. Lee, Y. et al., The nuclear RNase III Drosha initiates microRNA processing. Nature, 2003, 425, 415-419.

17. Denli, A. M., Tops, B. B., Plasterk, R. H., Ketting, R. F. and Hannon, G. J., Processing of primary microRNAs by the microprocessor complex. Nature, 2004, 432, 231-235.

18. Park, M. Y., Wu, G., Gonzalez-Sulser, A., Vaucheret, H. and Poethig, R. S., Nuclear processing and export of microRNAs in Arabidopsis. Proc. Natl. Acad. Sci. USA, 2005, 102, 3691-369.

19. Olsen, P. H. and Ambros, V., The lin-4 regulatory RNA controls developmental timing in Caenorhabditis elegans by blocking LIN14 protein synthesis after the initiation of translation. Dev. Biol., 1999, 216, 671-680.

20. Hammond, S. M., Boettcher, S., Caudy, A. A., Kobayashi, R. and Hannon, G. J., Argonaute2, a link between genetic and biochemical analyses of RNAi. Science, 2001, 293, 1146-1150.

21. Voinnet, O., Origin, biogenesis, and activity of plant microRNAs. Cell, 2009, 136, 669-687.

22. Iwasaki, S. et al., Hsc70/Hsp90 chaperone machinery mediates ATP-dependent RISC loading of small RNA duplexes. Mol. Cell, 2010, 39, 292-299.

23. Nakanishi, K., Anatomy of RISC: how do small RNAs and chaperones activate Argonaute proteins? Wiley Interdiscip. Rev. RNA, 2016, 7, 637-660.

24. Schwab, R., Ossowski, S., Riester, M., Warthmann, N. and Weigel, D., Highly specific gene silencing by artificial microRNAs in Arabidopsis. Plant Cell, 2006, 18, 1121-1133.

25. Fahim, M., Millar, A. A., Wood, C. C. and Larkin, P. J., Resistance to Wheat streak mosaic virus generated by expression of an artificial polycistronic microRNA in wheat. Plant Biotechnol. J., 2012, 10, 150-163.

26. Djami-Tchatchou, A. T., Sanan-Mishra, N., Ntushelo, K. and Dubery, I. A., Functional roles of microRNAs in agronomically important plants-potential as targets for crop improvement and protection. Front Plant Sci., 2017, 8, 378 .

27. Ding, J., Zhou, S. and Guan, J., Finding microRNA targets in plants: current status and perspectives. Genomics, Proteomics Bioinformat., 2012, 10, 264-275.

28. Spaink, H. P., Root nodulation and infection factors produced by rhizobial bacteria. Annu. Rev. Microbiol., 2000, 54, 257-288.

29. Harrison, M. J., Signaling in the arbuscular mycorrhizal symbiosis. Annu. Rev. Microbiol., 2005, 59, 19-42.

30. Patriarca, E. J., Tat'e, R., Ferraioli, S. and Iaccarino, M., Organogenesis of legume root nodules. Int. Rev. Cytol., 2004, 234, 201262

31. Simon, S. A., Meyers, B. C. and Sherrier, D. J., MicroRNAs in the rhizobia legume symbiosis. Plant Physiol., 2009, 151, 10021008 .

32. Combier, J. P. et al., MtHAP2-1 is a key transcriptional regulator of symbiotic nodule development regulated by microRNA169 in Medicago truncatula. Genes Dev., 2006, 20, 3084-3088.

33. Boualem, A. et al., MicroRNA166 controls root and nodule development in Medicago truncatula. Plant J., 2008, 54, 876887

34. Subramanian, S., Fu, Y., Sunkar, R., Barbazuk, W. B., Zhu, J. K. and $\mathrm{Yu}, \mathrm{O}$., Novel and nodulation-regulated microRNAs in soybean roots. BMC Genomics, 2008, 9, 160

35. Kasschau, K. D., Xie, Z., Allen, E., Llave, C., Chapman, E. J., Krizan, K. A. and Carrington, J. C., P1/HC-Pro, a viral suppressor of RNA silencing, interferes with Arabidopsis development and miRNA function. Dev. Cell, 2003, 4, 205-217.

36. Mallory, A. C., Bartel, D. P. and Bartel, B., MicroRNA-directed regulation of Arabidopsis AUXIN RESPONSE FACTOR17 is essential for proper development and modulates expression of early auxin response genes. Plant Cell, 2005, 17, 1360-1375.
37. Ryu, H., Cho, H., Choi, D. and Hwang, I., Plant hormonal regulation of nitrogen-fixing nodule organogenesis. Mol. Cells, 2012, 34, 117-126.

38. Champion, A. et al., Inhibition of auxin signaling in Frankiainfected cells in Casuarina glauca nodules leads to increased nodulation. Plant Physiol., 2015, 167, 1149-1157.

39. Yan, Z. et al., miR172 regulates soybean nodulation. Mol. PlantMicrobe Interact., 2013, 26, 1371-1377.

40. Nova-Franco, B. et al., The micro-RNA172c-APETALA2-1 node as a key regulator of the common bean-Rhizobium etli nitrogen fixation symbiosis. Plant Physiol., 2015, 168, 273-291.

41. Turner, M. et al., Ectopic expression of miR160 results in auxin hypersensitivity, cytokinin hyposensitivity, and inhibition of symbiotic nodule development in soybean. Plant Physiol., 2013, 162, 2042-2055.

42. Yan, Z., Hossain, M. S., Arikit, S., Valdés-López, O., Zhai, J. and Wang, J., Identification of microRNAs and their mRNA targets during soybean nodule development: functional analysis of the role of miR393j-3p in soybean nodulation. New Phytol., 2015, 207, 748-759.

43. Branscheid, A. et al., Expression pattern suggests a role of MiR399 in the regulation of the cellular response to local $\mathrm{Pi}$ increase during arbuscular mycorrhizal symbiosis. Mol. PlantMicrobe Interact., 2010, 23, 915-926.

44. Devers, E. A., Branscheid, A., May, P. and Krajinski, F., Stars and symbiosis: microRNA- and microRNA*-mediated transcript cleavage involved in arbuscular mycorrhizal symbiosis. Plant Physiol., 2011, 156, 1990-2010.

45. Lauressergues, D. et al., The microRNA miR171h modulates arbuscular mycorrhizal colonization of Medicago truncatula by targeting NSP2. Plant J., 2012, 72, 512-522.

46. Wu, P. et al., Identification of arbuscular mycorrhiza (AM)responsive microRNAs in tomato. Front Plant Sci, 2016, 7, 429.

47. Couzigou, J. M., Lauressergues, D., André, O., Gutjahr, C., Guillotin, B., Bécard, G. and Combier, J. P., Positive gene regulation by a natural protective miRNA enables arbuscular mycorrhizal symbiosis. Cell Host Microbe., 2016, 21, 106-112.

48. Ye, W., Shen, C. H., Lin, Y., Chen, P. J., Xu, X., Oelmüller, R., Yeh, K. W. and Lai, Z., Growth promotion-related miRNAs in Oncidium orchid roots colonized by the endophytic fungus Piriformospora indica. PLoS ONE, 2014, 9, e84920.

49. Fei, Q., Zhang, Y., Xia, R. and Meyers, B. C., Small RNAs add zing to the zig-zag-zig model of plant defenses. Mol. Plant Microbe Interact., 2016, 29, 165-169.

50. Chen, L., Luan, Y. and Zhai, J., Sp-miR396a-5p acts as a stressresponsive genes regulator by conferring tolerance to abiotic stresses and susceptibility to Phytophthora nicotianae infection in transgenic tobacco. Plant Cell Rep., 2015, 34, 2013-2025.

51. Boccara, M., Sarazin, A., Thiébeauld, O., Jay, F., Voinnet, O., Navarro, L. and Colot, V., The Arabidopsis miR472-RDR6 silencing pathway modulates PAMP- and effector-triggered immunity through the post-transcriptional control of disease resistance genes. PLoS Pathog., 2014, 10(1), e1003883.

52. Park, Y. J., Lee, H. J., Kwak, K. J., Lee, K., Hong, S. W. and Kang, H., MicroRNA400-guided cleavage of pentatricopeptide repeat protein mRNAs renders Arabidopsis thaliana more susceptible to pathogenic bacteria and fungi. Plant Cell Physiol., 2014, 55, 1660-1668.

53. Niu, D. et al., Bacillus cereus AR156 primes induced systemic resistance by suppressing miR825/825* and activating defenserelated genes in Arabidopsis. J. Integr. Plant Biol., 2016, 58, 426439.

54. Nie, P., Chen, C., Yin, Q., Jiang, C., Guo, J., Zhao, H. and Niu, D., Function of miR825 and miR825* as negative regulators in Bacillus cereus AR156-elicited systemic resistance to Botrytis cinerea in Arabidopsis thaliana. Int. J. Mol. Sci., 2019, 20, 5032; https://doi.org/10.3390/ijms20205032. 


\section{REVIEW ARTICLES}

55. Feng, H. et al., Identification of microRNAs and their corresponding targets involved in the susceptibility interaction of wheat response to Puccinia striiformis f. sp. tritici. Physiol. Plant., 2016, 157, 95-107.

56. Feng, H. et al., Target of tae-miR408, a chemocyanin-like protein gene $(T a C L P 1)$, plays positive roles in wheat response to highsalinity, heavy cupric stress and stripe rust. Plant Mol. Biol., 2013, 83, 433-443.

57. Hewezi, T., Piya, S., Qi, M., Balasubramaniam, M., Rice, J. H. and Baum, T. J., Arabidopsis miR827 mediates post-transcriptional gene silencing of its ubiquitin E3 ligase target gene in the syncytium of the cyst nematode Heterodera schachtii to enhance susceptibility. Plant J., 2016, 88, 179-192.

58. Li, D. et al., MicroRNA-mediated susceptible poplar gene expression regulation associated with the infection of virulent Melampsora larici-populina. BMC Genomics, 2016, 17, 59.

59. Islam, W., Noman, A., Qasim, M. and Wang, L., Plant responses to pathogen attack: small RNAs in focus. Int. J. Mol. Sci., 2018, 19, 515 .

60. Navarro, L. et al., A plant miRNA contributes to antibacterial resistance by repressing auxin signaling. Science, 2006, 312, 436439.

61. Robert-Seilaniantz, A., MacLean, D., Jikumaru, Y., Hill, L., Yamaguchi, S., Kamiya, Y. and Jones, J. D., The microRNA miR393 re-directs secondary metabolite biosynthesis away from camalexin and towards glucosinolates. Plant J., 2011, 67, 218231

62. Bian, H. et al., Distinctive expression patterns and roles of the miRNA393/TIR1 homolog module in regulating flag leaf inclination and primary and crown root growth in rice (Oryza sativa). New Phytol, 2012, 196, 149-161.

63. Xia, K. et al., OsTIR1 and OsAFB2 down-regulation via OsmiR393 overexpression leads to more tillers, early flowering and less tolerance to salt and drought in rice. PLOS ONE, 2012, 7, e30039.

64. Pruss, G. J., Nester, E. W. and Vance, V., Infiltration with Agrobacterium tumefaciens induces host defense and developmentdependent responses in the infiltrated zone. Mol. Plant Microbe Interact., 2008, 21, 1528-1538.

65. Zhang, X. et al., Arabidopsis Argonaute 2 regulates innate immunity via miRNA393 (*)-mediated silencing of a Golgi localized SNARE gene, MEMB12. Mol. Cell, 2011, 42, 356-366.

66. Fahlgren, N. et al., High-throughput sequencing of Arabidopsis microRNAs: evidence for frequent birth and death of MIRNA genes. PLoS ONE, 2007, 2, e219.

67. Li, Y., Zhang, Q., Zhang, J., Wu, L., Qi, Y. and Zhou, J. M., Identification of microRNAs involved in pathogen-associated molecular pattern triggered plant innate immunity. Plant Physiol., 2010, 152, 2222-2231.

68. Sunkar, R. and Zhu, J. K., Novel and stress-regulated microRNAs and other small RNAs from Arabidopsis. Plant Cell, 2004, 16, 2001-2019.

69. Jagadeeswaran, G., Saini, A. and Sunkar, R., Biotic and abiotic stress down-regulate miR398 expression in Arabidopsis. Planta, 2009, 229, 1009-1014.

70. He, X. F., Fang, Y. Y., Feng, L. and Guo, H. S., Characterization of conserved and novel microRNAs and their targets, including a
TuMV-induced TIR-NBS-LRR class $\mathrm{R}$ gene-derived novel miRNA in Brassica. FEBS Lett., 2008, 582, 2445-2352.

71. Du, P. et al., Viral infection induces expression of novel phased microRNAs from conserved cellular microRNA precursors. PLoS Pathog., 2011, 7, e1002176.

72. Wu, J. et al., Viral inducible Argonaute 18 confers broad-spectrum virus resistance in rice by sequestering a host microRNA. eLife, $2015,4, \mathrm{e} 05733$.

73. Shivaprasad, P. V., Chen, H. M., Patel, K., Bond, D. M., Santos, B. A. and Baulcombe, D. C., A microRNA superfamily regulates nucleotide binding site leucine-rich repeats and other mRNAs. Plant Cell, 2012, 24, 859-874.

74. Sun, L. et al., Dimeric artificial microRNAs mediate high resistance to RSV and RBSDV in transgenic rice plants. Plant Cell Tissue Organ Cult., 2016, 126, 127-139.

75. Li, Y. et al., Multiple rice microRNAs are involved in immunity against the blast fungus Magnaporthe oryzae. Plant Physiol., 2013, 164, 1077-1092.

76. Campo, S. et al., Identification of a novel microRNA (miRNA) from rice that targets an alternatively spliced transcript of the Nramp6 (Natural resistance-associated macrophage protein 6) gene involved in pathogen resistance. New Phytol., 2013, 199, $212-227$.

77. Fones, H. and Preston, G. M., The impact of transition metals on bacterial plant disease. FEMS Microbiol. Rev., 2013, 37, 495519.

78. Gupta, O. P., Permar, V., Koundal, V., Singh, U. D. and Praveen, S., MicroRNA regulated defense responses in Triticum aestivum L. during Triticum aestivum f. sp. tritici infection. Mol. Biol. Rep., 2012, 39, 817-824.

79. Guo, H. S., Xie, Q., Fei, J. F. and Chua, N. H., MicroRNA directs mRNA cleavage of the transcription factor $N A C 1$ to down regulate auxin signals for Arabidopsis lateral root development. Plant Cell, 2005, 17, 1376e86.

80. Xin, M. et al., Diverse set of microRNAs are responsive to powdery mildew infection and heat stress in wheat (Triticum aestivum L.). BMC Plant Biol., 2010, 10, 123.

81. Manickavelu, A. et al., Comprehensive functional analyses of expressed sequence tags in common wheat (Triticum aestivum). DNA Res., 2012, 19, 165-177.

82. Luan, Y., Cui, J., Li, J., Jiang, N., Liu, P. and Meng, J., Effective enhancement of resistance to Phytophthora infestans by overexpression of miR172a and b in Solanum lycopersicum. Planta, 2018, 247, 127-138.

83. Zhang, T. et al., Cotton plants export microRNAs to inhibit virulence gene expression in a fungal pathogen. Nature Plants, 2016, 2, 16153.

ACKNOWLEDGEMENTS. We thank the Central University of Kerala, Kasargod for providing the necessary facilities. M.M.N. is grateful to DST, New Delhi for INSPIRE fellowship support.

Received 1 February 2019; revised accepted 12 August 2020

doi: $10.18520 / \mathrm{cs} / \mathrm{v} 119 / \mathrm{i} 8 / 1282-1290$ 